\title{
Sistema de gestión de calidad del programa educativo de ingeniería Industrial de la UABC
}

\author{
Quality Management System of the Industrial Engineering Educational \\ Program of the UABC
}

Sistema de gestão da qualidade do programa de Engenharia Industrial da

UABC

Edgar Armando Chávez Moreno

Universidad Autónoma de Baja California, México

gared74mx@uabc.edu.mx https://orcid.org/0000-0002-9305-3595

\section{Resumen}

Esta investigación cualitativa presenta los resultados de una autoevaluación realizada en abril de 2020 para medir el sistema de gestión de calidad del programa educativo de ingeniería Industrial de la Facultad de Ciencias de la Ingeniería y Tecnología de la Universidad Autónoma de Baja California. Se utilizó la metodología de los Comités Interinstitucionales para la Evaluación de la Educación Superior (Ciees) y una escala de Likert. Los resultados muestran que el programa en cuestión cumple con un nivel suficiente, adecuado u óptimo en $95 \%$ de los estándares de los Ciees, reflejo de un buen sistema de gestión de calidad. Las conclusiones confirman que la acreditación de un programa educativo por parte de organismos externos garantiza la calidad educativa.

Palabras clave: calidad educativa, educación superior, ingeniería industrial. 


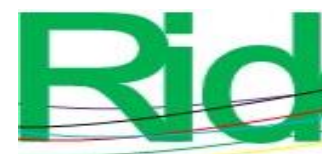

\section{Abstract}

This qualitative research presents the results of a self-assessment carried out in April 2020 to measure the quality management system of the Industrial Engineering educational program of the Faculty of Engineering Sciences and Technology of the Universidad Autónoma de Baja California. The methodology of the Comités Interinstitucionales para la Evaluación de la Educación Superior (CIEES) and a Likert scale were used. The results show that the program in question meets a sufficient, adequate or optimal level in $95 \%$ of the CIEES standards, a reflection of a good quality management system. The conclusions confirm that the accreditation of an educational program by external organizations guarantees educational quality.

Keywords: educational quality, higher education, industrial engineering.

\section{Resumo}

Esta pesquisa qualitativa apresenta os resultados de uma autoavaliação realizada em abril de 2020 para medir o sistema de gestão da qualidade do programa educacional de Engenharia Industrial da Faculdade de Ciências e Tecnologia de Engenharia da Universidad Autónoma de Baja California. Foi utilizada a metodologia dos Comités Interinstitucionales para la Evaluación de la Educación Superior (Ciees) e escala Likert. Os resultados mostram que o programa em questão atende a um nível suficiente, adequado ou ótimo em $95 \%$ dos padrões do Ciees, reflexo de um bom sistema de gestão da qualidade. As conclusões confirmam que o credenciamento de um programa educacional por organizações externas garante a qualidade educacional.

Palavras-chave: qualidade educacional, ensino superior, engenharia industrial.

Fecha Recepción: Noviembre 2020

Fecha Aceptación: Julio 2021 


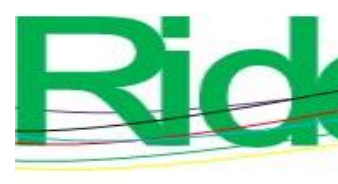

Revista Iberoamericana para la Investigación y el Desarrollo Educativo ISSN $2007-7467$

\section{Introducción}

Debido a los avances en el área de las tecnologías de la información y la comunicación (TIC), así como a los cambios organizacionales, políticos, económicos, sociales y a las demandas del mercado, la calidad educativa en la educación superior es un tema que ha venido adquiriendo gran relevancia a nivel mundial. Según estudios recientes de la Organización para la Cooperación y el Desarrollo Económicos [OCDE] (2019), en México, al igual que en el resto de los países miembros de la OCDE, las personas que logran obtener un grado académico universitario tienen mayores probabilidades de conseguir un trabajo mejor remunerado, lo que incrementa su nivel de calidad de vida.

Respecto a cómo medir la calidad en el sector educativo de nivel superior, la Asociación Nacional de Universidades e Instituciones de Educación Superior [Anuies] (2018) menciona tres maneras de hacerlo. Una de ellas apunta a la eficacia de la acción educativa, es decir, al logro de los aprendizajes esperados en un periodo de tiempo. Otra se refiere a la relevancia de los contenidos de aprendizaje y cómo estos satisfacen las expectativas y necesidades de los interesados para su desarrollo y desempeño profesional. También puede concebirse a partir de los insumos utilizados y los procesos de la experiencia educativa, es decir, con base en la organización, la normativa, los planes y programas de estudio, las capacidades de la planta docente, la infraestructura y el equipamiento (Anuies, 2018).

Para Delahoz, Guillen y Fontalvo (2020), es importante que los programas educativos cuenten con una acreditación de calidad, sin importar el criterio utilizado, ya que influye de manera significativa en el desempeño de los estudiantes universitarios. Y en esa misma línea, la Asociación Europea para la Calidad de la Educación Superior [EQNA, por sus siglas en inglés] (2015) refiere que debe buscarse un aseguramiento interno de calidad a través de mecanismos que permitan operar de manera eficiente los procesos de enseñanza-aprendizaje, y que una manera de sostener una mejora continua de calidad es mediante las acreditaciones a los programas educativos que realizan organismos externos.

Para el presente estudio, se toma como referencia el sistema de gestión de calidad implementado al interior del programa educativo de ingeniería Industrial de la Facultad de Ciencias de la Ingeniería y Tecnología (FCITEC) de la Universidad Autónoma de Baja California (UABC) (León et al., 2018; Moreno, 2018). Dicho programa se sometió a los 95 estándares de calidad que establecen los Comités Interinstitucionales para la Evaluación de 


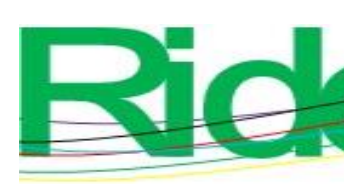

Revista Iberoamericana para la
Investigación y el Desarrollo Educativo
ISSN $2007-7467$

la Educación Superior (Ciees), máximo organismo en México que acredita la calidad educativa de los programas de estudio en las instituciones de educación superior.

\section{La calidad educativa en el nivel superior}

Uno de los documentos más interesantes y significativos para los fines de este estudio es el Plan de Desarrollo Institucional 2019-2023 de la UABC (2019), que describe el panorama educativo presente en México, así como las tendencias a nivel mundial en materia de calidad y equidad. Siguiendo lo señalado por la Organización para la Cooperación y el Desarrollo Económicos (2019), la UABC (2019) planteó que uno de los temas más importantes para mejorar la educación superior es el establecimiento de políticas de aseguramiento de calidad.

Cabe señalar que, al igual que la OCDE, la Organización de las Naciones Unidas para la Educación, la Ciencia y la Cultura (Unesco), desde la Conferencia Mundial de 1998, ha subrayado la importancia de la calidad en la educación superior, así como ha impulsado el establecimiento de cambios significativos en pro de la equidad en la oferta educativa por parte de las instituciones de educación superior. Al respecto, la Organización de las Naciones Unidas (ONU) (Asamblea General, 2015), dentro de la Agenda 2030 para el Desarrollo Sostenible, se ha propuesto lo siguiente: "Para 2030, asegurar el acceso en condiciones de igualdad para todos los hombres y las mujeres a una formación técnica, profesional y superior de calidad, incluida la enseñanza universitaria" (p. 19). Asimismo, el objetivo cuatro declara: "Garantizar una educación inclusiva, equitativa y de calidad y promover oportunidades de aprendizaje durante toda la vida para todos" (p. 19).

Volviendo al Plan de Desarrollo Institucional 2019-2023 (UABC, 2019), aquí se menciona de manera textual lo siguiente:

En el futuro próximo la educación superior ha de considerar las siguientes tendencias: $a$ ) expansión de los sistemas de educación superior; $b$ ) cobertura, calidad y equidad; c) transformación del mundo del trabajo; $d$ ) indicaciones de desempeño como mecanismo para la rendición de cuentas, y e) ciudadanía, responsabilidad social y derechos humanos (p. 15). 


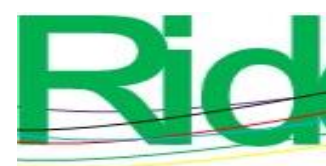

Revista Iberoamericana para la Investigación y el Desarrollo Educativo ISSN 2007 - 7467

\section{Metodología}

Este trabajo es un estudio de caso cualitativo y descriptivo (Martínez, 2006). Se emprendió con el fin de identificar la calidad educativa y establecer una cultura de mejora continua en el programa educativo de ingeniería Industrial de la FCITEC de la UABC. Se toma como referencia la metodología de los Ciees (2018), la cual incluye cinco ejes, 12 categorías, 49 indicadores y 95 estándares que miden la calidad educativa, como se muestra en la figura 1.

Figura 1. Ejes y categorías para la evaluación del PEII

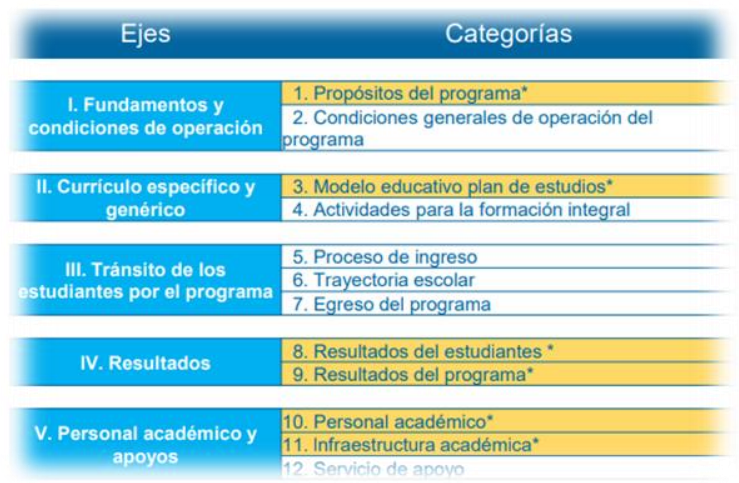

Fuente: Ciees (2018)

\section{Resultados}

La autoevaluación fue realizada por un grupo de siete académicos de la FCITEC (figura 2) y en función de las evidencias recabadas del sistema de gestión de calidad del programa educativo de ingeniería Industrial. Para trabajar los 95 estándares de los 45 indicadores, en las 12 categorías de los cinco ejes de la metodología del Ciees, se formuló la siguiente escala: No aplica (NA), Insuficiente (Ins), Suficiente (Suf), Adecuado (Ade) y Óptimo (Opt). Cabe señalar que los datos obtenidos se manejaron con apoyo del paquete estadístico Minitab 17. Dichos resultados se presentan a continuación. 


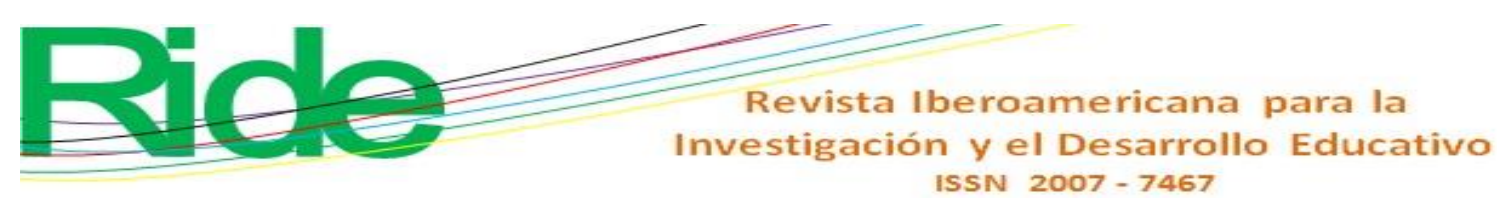

Figura 2. FCITEC de la UABC

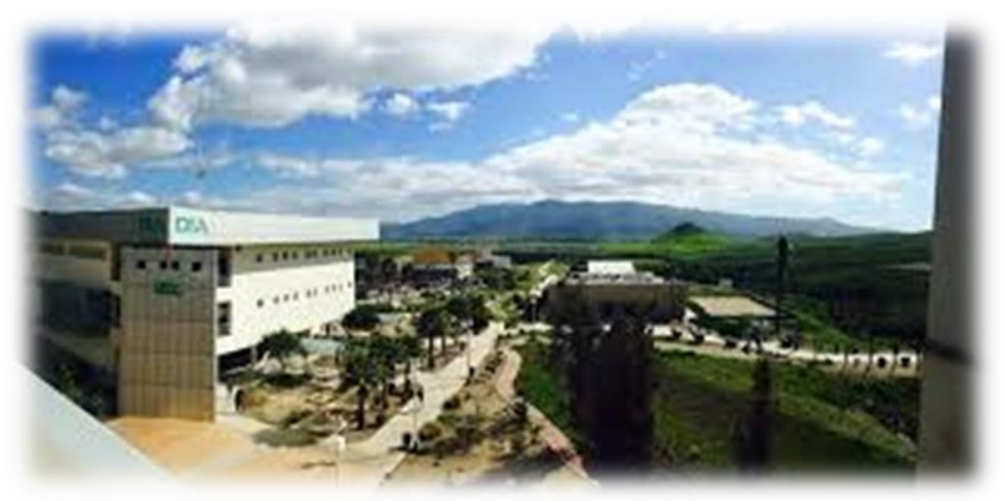

Fuente: Ingeniería Industrial UABC (s. f.)

En la figura 3 se puede observar que los 10 estándares de la categoría uno, "Propósitos del programa", obtuvieron una puntuación de Óptimo.

Figura 3. Autoevaluación de la categoría uno Ciees del programa de ingeniería Industrial de la FCITEC

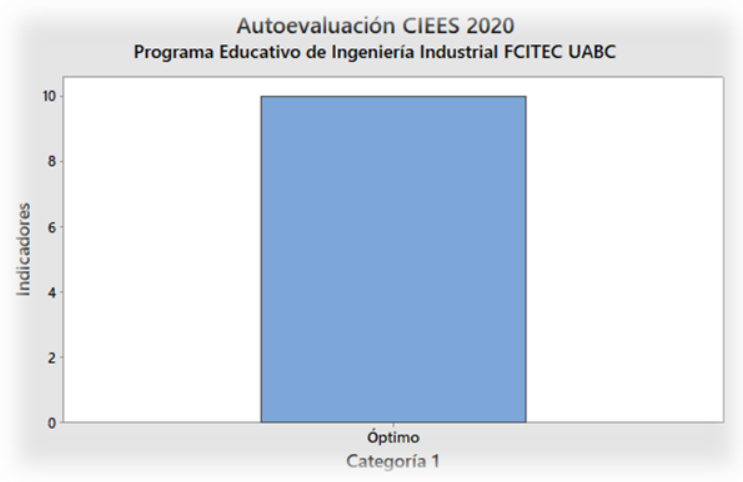

Fuente: Elaboración propia

Dentro de las fortalezas que se identificaron se encuentran las tutorías académicas, el cumplimiento de los lineamientos de los comités acreditadores de la enseñanza de la ingeniería, el servicio social profesional y la vinculación con el sector productivo mediante estadías de aprendizaje. Mientras que se observaron las siguientes debilidades: en el plan de estudios se recomienda consolidar el área económico-financiera y una seriación de las materias en el plan de estudios 2009-2.

Dentro de la categoría dos, "Condiciones generales de operación del programa", los cinco estándares obtuvieron la puntuación máxima, tal como se puede apreciar en la figura 4 . 

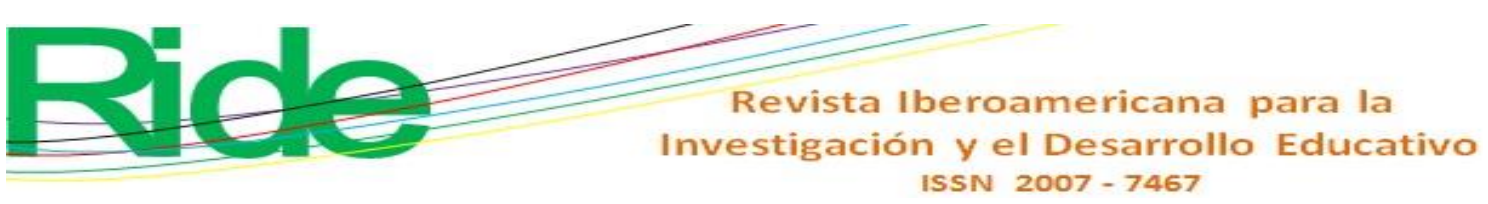

Figura 4. Autoevaluación de la categoría dos Ciees del programa de ingeniería Industrial de la FCITEC

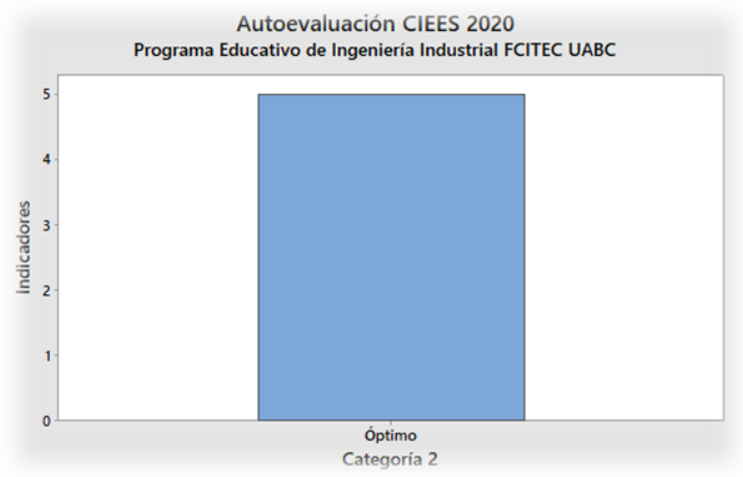

Fuente: Elaboración propia

Las fortalezas que se identificaron al interior del sistema de gestión de calidad del programa educativo fueron que cumple los requisitos que marca la Secretaría de Educación Pública (SEP), existe un orden y una normatividad del programa educativo y un crecimiento ligero pero gradual de la matrícula. Mientras que las debilidades detectadas fueron un posible presupuesto limitado y que requiere de una nueva estructura organizacional.

En la categoría tres, "Modelo educativo y plan de estudios", el sistema de gestión de calidad del programa educativo obtuvo Óptimo en seis estándares y en uno Adecuado, según la opinión de los siete académicos de la FCITEC.

Figura 5. Autoevaluación de la categoría tres Ciees del programa de ingeniería Industrial de la FCITEC

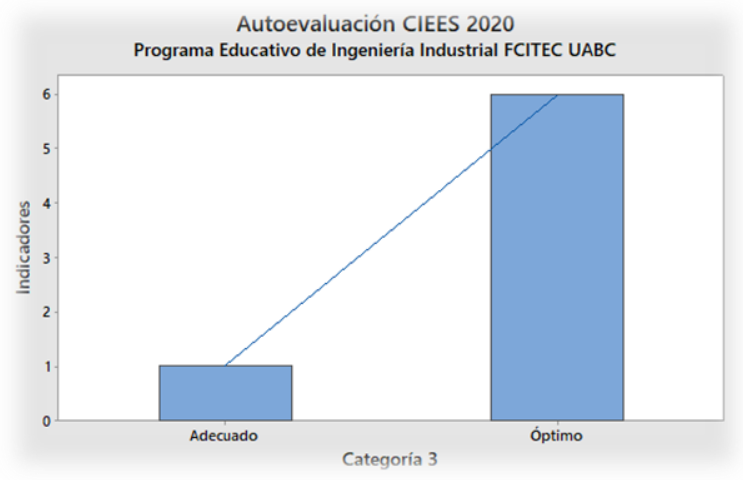

Fuente: Elaboración propia

Por un lado, las fortalezas encontradas en el sistema de gestión de calidad del programa educativo fueron el modelo educativo de la $\mathrm{UABC}$, un programa de capacitación docente permanente, un nuevo plan de estudios 2019-2, así como un instrumento de 


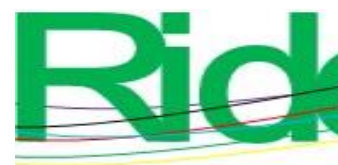

Revista Iberoamericana para la
Investigación y el Desarrollo Educativo
ISSN $2007-7467$

evaluación docente a disposición de los alumnos. Por el otro, las debilidades que se identificaron fueron que algunos profesores de asignatura no han llevado el curso de Inducción y la necesidad de implementar un mecanismo para verificar el cumplimiento de las competencias y los contenidos temáticos.

En cuanto a la categoría cuatro, "Actividades para la formación integral”, tal y como se muestra en la figura 6, el sistema de gestión de calidad del programa educativo presentó una mayor variedad de notas: un estándar con Insuficiente, otro con Suficiente, dos estándares fueron calificados con Adecuado y tres estándares con un Óptimo.

Figura 6. Autoevaluación de la categoría cuatro Ciees del programa de ingeniería Industrial de la FCITEC

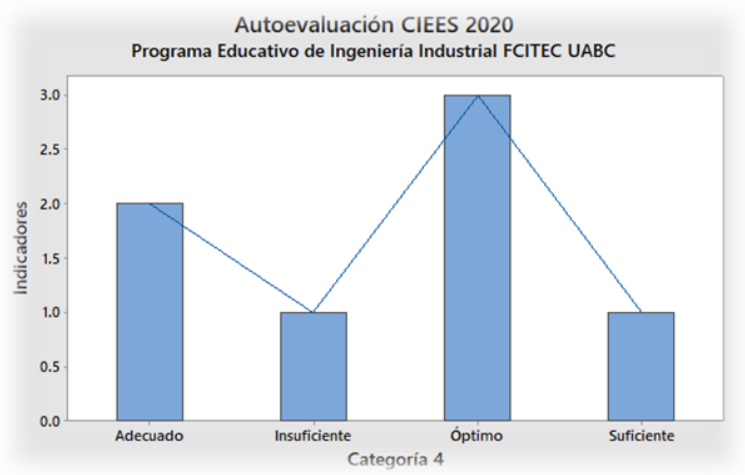

Fuente: Elaboración propia

Las fortalezas que se encontraron dentro de esta categoría fueron que los estudiantes presentan una formación integral, se cuenta con apoyos para desarrollar un segundo idioma por parte de la Facultad de Idiomas y de la propia FCITEC, se cuenta con una red de valores institucionales y se oferta la certificación de SolidWorks. Como debilidades se identificaron que existe poca promoción de las actividades extracurriculares y que se requiere ampliar la oferta de certificaciones para los estudiantes del programa.

Respecto a la categoría cinco, "Proceso de ingreso", los académicos puntuaron dos estándares con Suficiente y los otros dos con Óptimo, tal como se puede apreciar en la figura 7. 

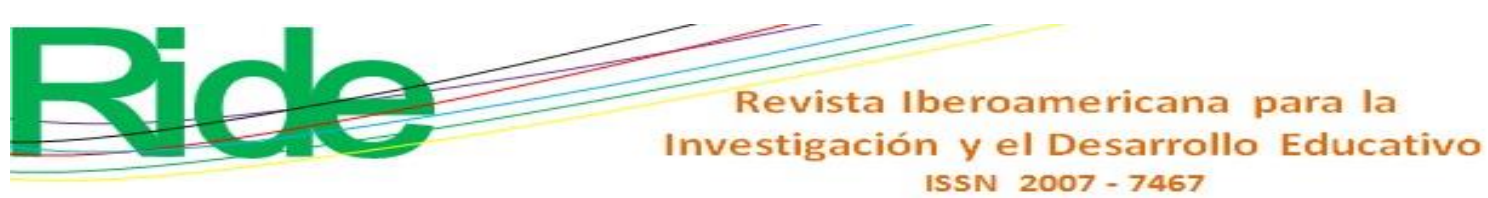

Figura 7. Autoevaluación de la categoría cinco Ciees del programa de ingeniería Industrial de la FCITEC

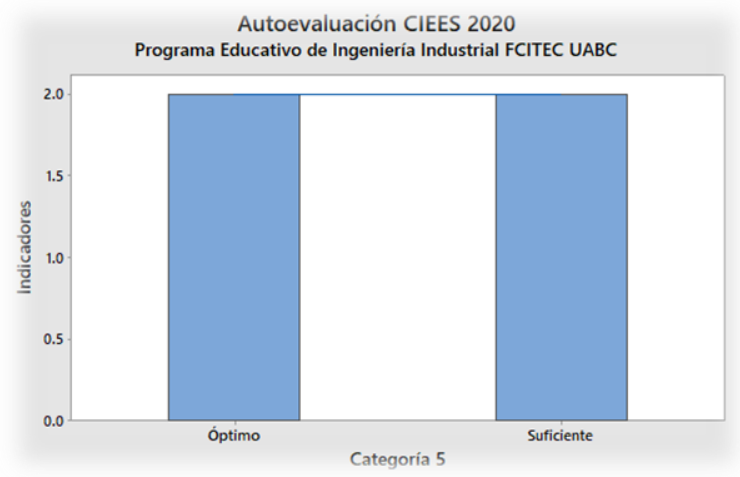

Fuente: Elaboración propia

Aquí se logró identificar como fortalezas la bienvenida a los estudiantes de nuevo ingreso, las actividades de inducción para que los estudiantes de nuevo ingreso se integren de manera informada, el curso de nivelación académica (CNA) en matemáticas y el programa de asesorías académicas. Como debilidades se identificaron la necesidad de establecer un seguimiento del CNA y la falta de seguimiento del programa de asesorías.

En la categoría "Trayectoria escolar", tres estándares alcanzaron un Suficiente, tres fueron calificadas como Adecuado y dos estándares la nota de Óptimo.

Figura 8. Autoevaluación de la categoría seis Ciees del programa de ingeniería Industrial de la FCITEC

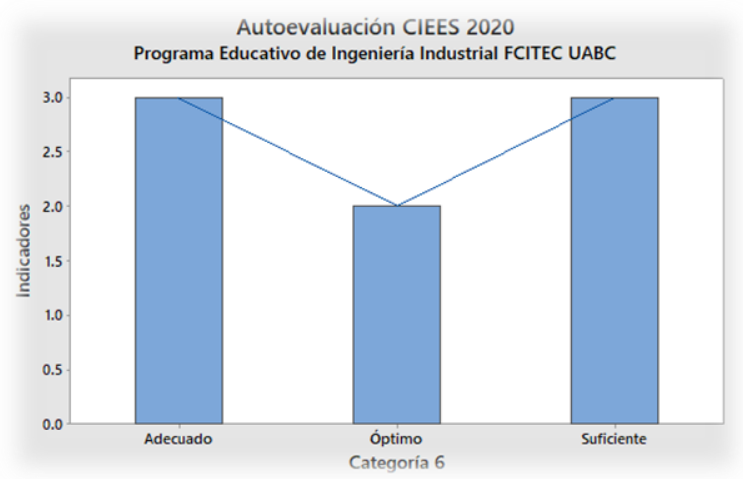

Fuente: Elaboración propia

En este caso, las principales fortalezas encontradas fueron las prácticas profesionales, servicio social profesional y proyectos de vinculación con valor en créditos, el programa de becas para alumnos y el programa de asesorías y tutorías para disminuir los índices de reprobación, rezago y deserción escolar. Mientras que la única debilidad observada fue la 


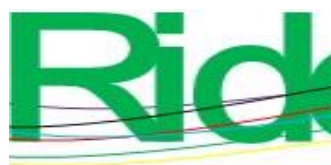

Revista Iberoamericana para la
Investigación y el Desarrollo Educativo
ISSN $2007-7467$

necesidad de implementar un instrumento que cuantifique el impacto final de los alumnos que asisten a tutorías o asesorías sobre el índice de deserción y rezago.

La figura 9, por su parte, muestra las puntuaciones obtenidas para los estándares de la categoría 7, "Egreso del programa".

Figura 9. Autoevaluación de la categoría siete Ciees del programa de ingeniería Industrial de la FCITEC

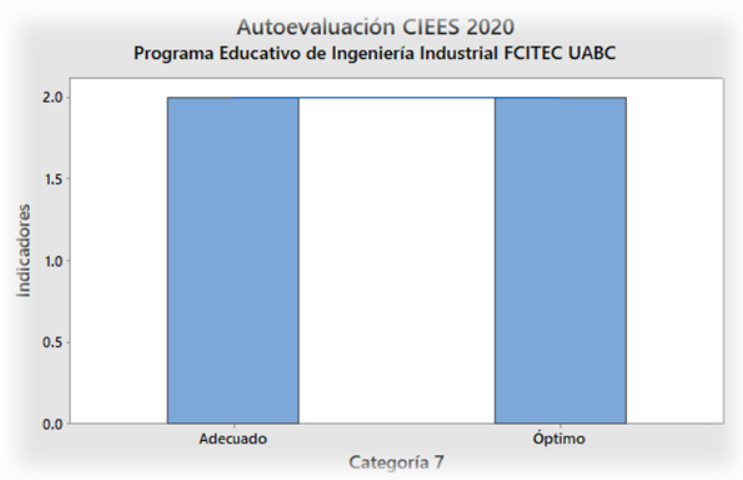

Fuente: Elaboración propia

Las fortalezas encontradas, a partir de la autoevaluación en esta categoría, fueron las siguientes: el programa educativo, a través de la UABC, ofrece a los egresados diversas opciones para obtener su título de ingeniero Industrial y la carrera, a través de la UABC, cuenta con un programa que orienta a los egresados en su transición de estudiantes a profesionistas. Por su parte, las debilidades observadas tuvieron que ver con el hecho de que la tasa de titulación de los estudiantes del programa educativo es baja y la bolsa de trabajo de la $\mathrm{UABC}$ es institucional, incluye a todos los programas educativos que se ofertan, y no es específica para el programa de ingeniería Industrial.

La figura 10 muestra los niveles alcanzados por los estándares de la categoría ocho, "Resultados de los estudiantes", donde un estándar alcanzó un Insuficiente, un estándar el nivel Suficiente, dos estándares obtuvieron una puntuación Adecuado y cuatro un nivel Óptimo. 

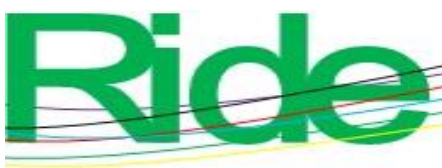

Revista Iberoamericana para la Investigación y el Desarrollo Educativo ISSN $2007-7467$

Figura 10. Autoevaluación de la categoría ocho Ciees del programa de ingeniería Industrial de la FCITEC

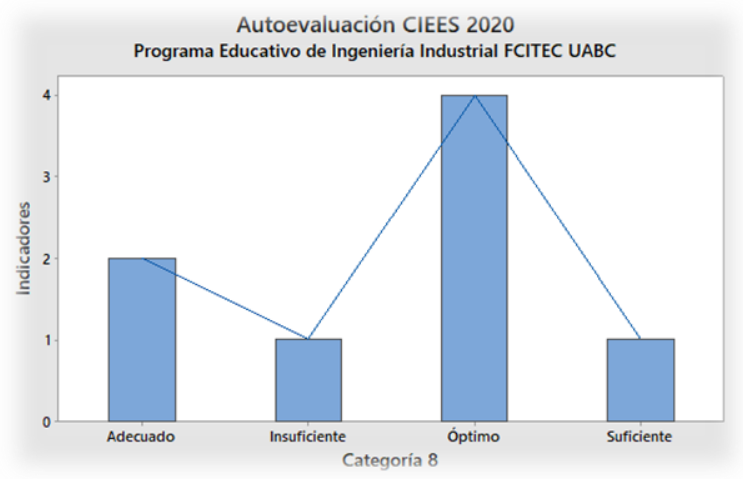

Fuente: Elaboración propia

Las fortalezas identificadas fueron que se fomenta la participación activa de los estudiantes en concursos y foros de importancia nacional e internacional, se cuenta con un examen de egreso externo que permite evaluar de forma estandarizada el conocimiento de los egresados, los empleadores reconocen la capacidad de resolver problemas de los egresados y se cuenta con un programa de movilidad estudiantil, mediante el cual los estudiantes pueden viajar al extranjero y aprender o perfeccionar un segundo idioma. Las debilidades observadas fueron que existen pocas certificaciones en la facultad, los resultados del Examen General para el Egreso de Licenciatura (EGEL) son poco satisfactorios, los índices de eficiencia terminal y de titulación son bajos y existe poco dominio de un segundo idioma.

Dentro de la categoría nueve, "Resultados del programa", dos estándares obtuvieron un Insuficiente, un estándar alcanzó la nota Suficiente y seis estándares la de Óptimo, tal como se puede apreciar en la figura 11. 

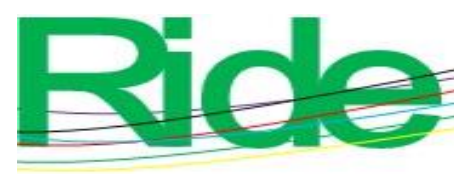

Revista Iberoamericana para la Investigación y el Desarrollo Educativo ISSN 2007 - 7467

Figura 11. Autoevaluación de la categoría nueve Ciees del programa de ingeniería Industrial de la FCITEC

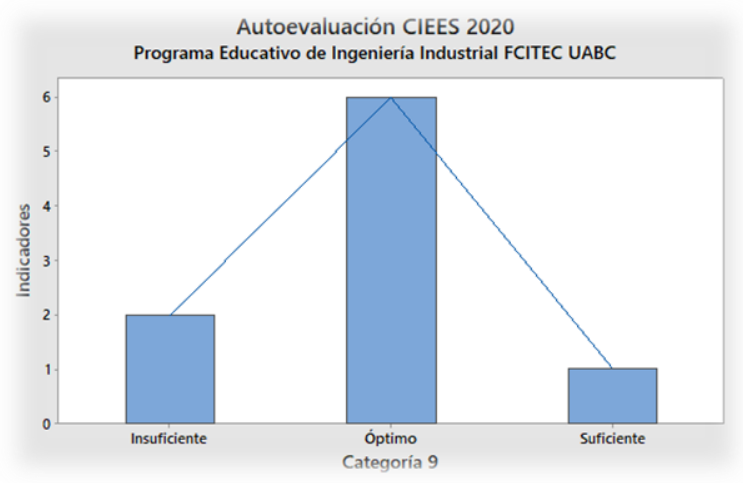

Fuente: Elaboración propia

Aquí las fortalezas encontradas en la autoevaluación en función del sistema de gestión de calidad del programa educativo fueron que el índice de deserción de los estudiantes es relativamente bajo, se cuenta con un programa de asesorías académicas para apoyar a los estudiantes de bajo rendimiento, los egresados logran incorporarse al sector laboral dentro de los primeros seis meses de egresar y los egresados se encuentran laborando en actividades directamente relacionadas con lo que estudiaron. Por otro lado, las debilidades del programa educativo detectadas en esta categoría fueron que la eficiencia de titulación es baja, hace falta mayor promoción de las certificaciones existentes y se deben establecer estrategias para dar seguimiento a los egresados que se encuentren realizando estudios de posgrado.

En cuanto a la categoría referente al personal académico, un estándar obtuvo una puntuación Suficiente, dos estándares un Adecuado y tres estándares un Óptimo, tal como se logra identificar en la figura 12. 


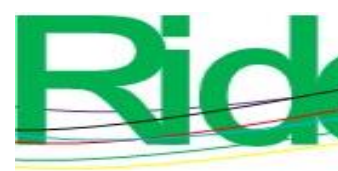

Revista Iberoamericana para la
Investigación y el Desarrollo Educativo
ISSN $2007-7467$

Dentro de las fortalezas identificadas en esta categoría, se encuentra la cantidad y calidad de aulas, laboratorios y talleres, que el mobiliario cuenta con la calidad necesaria realizar las diferentes actividades, los convenios establecidos con empresas y organismos gubernamentales, el acervo bibliográfico amplio y pertinente para la carrera y la capacidad tecnológica. Mientras que las debilidades encontradas van orientadas a que es recomendable que se tenga un plan de actualización de la infraestructura informática, tanto de equipos como de programas informáticos.

En relación con los estándares de la categoría 12, "Servicios de apoyo", uno de ellos obtuvo No aplica, dos estándares Adecuado y cinco fueron calificados como Óptimo (figura 14)

Figura 14. Autoevaluación de la categoría 12 Ciees del programa de ingeniería Industrial de la FCITEC

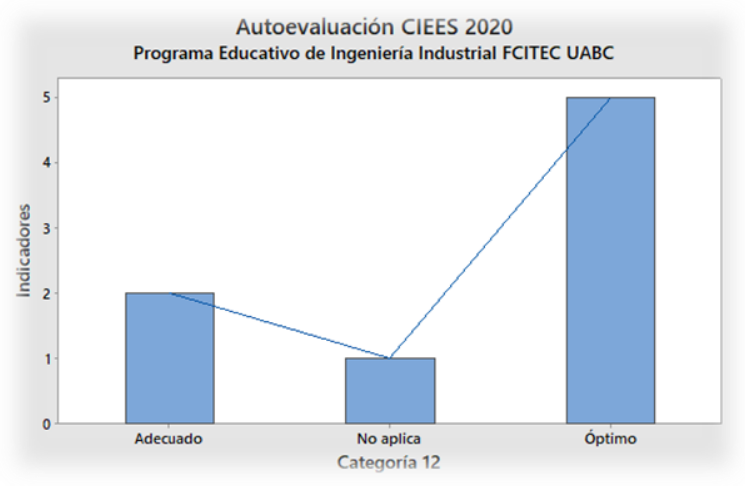

Fuente: Elaboración propia

El alumno puede realizar algunos trámites totalmente en línea, existe un programa de becas amplio y adecuado a las necesidades de los alumnos y se cuenta con un Departamento Psicopedagógico que brinda apoyo a los estudiantes de manera oportuna e integral, esas fueron las fortalezas detectadas. Mientras que las debilidades fueron que no se ha logrado garantizar un transporte eficiente para la comunidad de la unidad de Valle de las Palmas y que el Departamento Psicopedagógico no tiene evidencia del impacto en la mejora de la trayectoria escolar de los estudiantes que se han atendido.

Finalmente, se concentraron los 95 estándares de calidad de la metodología de los Ciees adaptados al programa educativo de la FCITEC de la UABC por los pares académicos en un diagrama de Pareto con apoyo de Minitab 17, y se obtuvo la nota de Suficiente en 94.7\% de los estándares de calidad, es decir, 90 de los 95 estándares cumple con los 

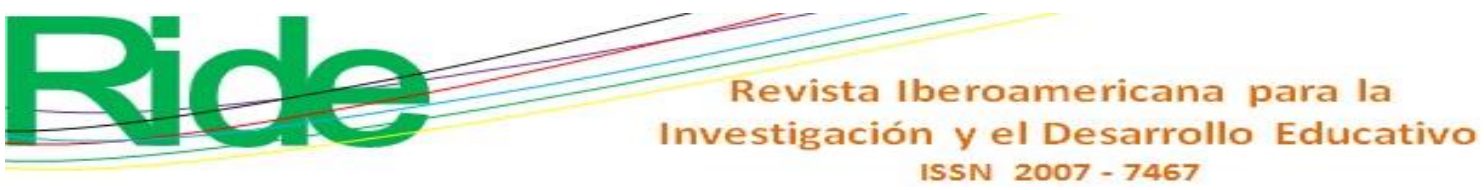

lineamientos establecidos por el organismo acreditador, por lo que, basándonos en estos resultados, puede aspirar a formar parte del Padrón Nacional de Calidad de Programas Educativos, tal como lo refleja la figura 15.

Figura 15. Diagrama de Pareto de la autoevaluación del programa de ingeniería Industrial de la FCITEC

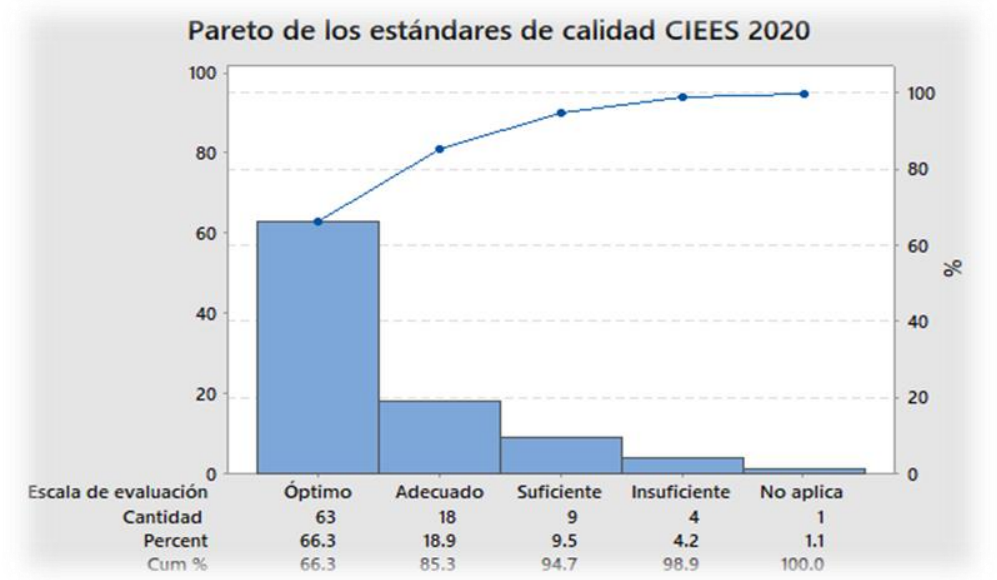

Fuente: Elaboración propia

Actualmente, el programa educativo de ingeniería Industrial se encuentra en espera del dictamen que emitirá los Ciees; de ser favorable, será un respaldo importante de que el sistema de gestión de calidad implementado garantiza una calidad educativa de calidad.

\section{Discusión}

Algunas de las limitaciones que se tuvieron en este estudio radican en el hecho de que no se contó con el dictamen final de los Ciees, que permitiera comparar la autoevaluación realizada por los pares académicos al interior del programa educativo de ingeniería Industrial del sistema de gestión de calidad con el proceso oficial del organismo acreditador.

En cuanto a las fortalezas de este estudio, se encuentra principalmente la disponibilidad y acceso a la información para llevar a cabo la investigación, así como un sistema de gestión de calidad en cierta medida validado al interior del programa educativo de ingeniería Industrial de la FCITEC, lo que coincide con el planteamiento de UABC (2019) que prepondera la calidad educativa. 


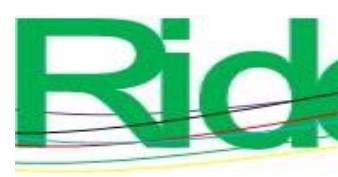

Revista Iberoamericana para la Investigación y el Desarrollo Educativo ISSN 2007 - 7467

Por otro lado, los resultados de este estudio refuerzan lo que otros estudios apuntan (EQNA, 2015): la forma de garantizar la calidad educativa de los estudiantes en las universidades es precisamente evaluando y acreditando los programas de estudios por organismos externos, tal como en este estudio se realizó bajo la metodología de los Ciees.

Finalmente, contar con recursos para poder llevar a cabo una investigación comparativa con otros programas de estudio de la propia universidad, o incluso de universidades extranjeras, hubiera producido más resultados que permitieran realizar una nueva propuesta de un sistema de gestión de calidad, alineado no solo con la metodología de los Ciees, sino con metodologías de otros organismos acreditadores, así como realizar un cuadro comparativo en el tema de calidad educativa.

\section{Conclusiones}

El contar con un sistema de gestión de calidad basado en los 95 estándares de la metodología de los Ciees ayudó, orientó y preparó al programa educativo de ingeniería Industrial de la FCITEC.

Dentro de la autoevaluación llevada a cabo por los pares académicos del programa educativo, los estándares de calidad de la categoría uno, "Propósitos del programa", categoría dos, "Condiciones generales de operación del programa", categoría tres, "Modelo educativo y plan de estudios", categoría seis, "Trayectoria escolar", categoría siete, "Egreso del programa", categoría 10, "Personal académico", categoría 11, "Infraestructura académica" y categoría 12, "Servicios de apoyo", cumplieron con la calidad establecida por los Ciees.

Por otro lado, dentro de los estándares de calidad de la categoría cuatro, “Actividades para la formación integral", se encontró un área de mejora en cuanto a la cantidad de certificaciones relacionadas con calidad, producción o manufactura, ya que son pocos los estudiantes que cuentan con este tipo de certificaciones. Dentro de los estándares de la categoría ocho, "Resultados de los estudiantes", se identificaron resultados por debajo de la media nacional en los egresados que presentaron el EGEL, un instrumento diseñado por el Centro Nacional de Evaluación para la Educación Superior (Ceneval) que mide el nivel del conocimiento en ingeniería industrial. En la categoría nueve, "Resultados del programa", se encontró como área de oportunidad mejorar el seguimiento de egresados al interior del programa educativo. 

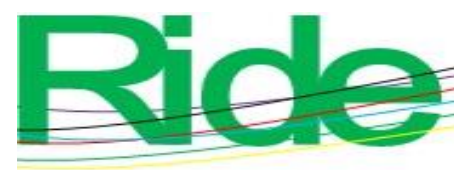

Revista Iberoamericana para la Investigación y el Desarrollo Educativo

ISSN $2007-7467$

Actualmente, el programa educativo de ingeniería industrial de la FCITEC de la UABC se encuentra esperando el dictamen de la evaluación remota llevada a cabo por los Ciees. Una vez emitido el dictamen, se podrán comparar dichos resultados del organismo externo con los de la autoevaluación llevada a cabo en este estudio, lo que deja abierta la invitación a otros investigadores interesados en llevar a cabo un estudio comparativo.

\section{Futuras líneas de investigación}

A partir de los resultados y hallazgos del proyecto de investigación, surgieron posibles vertientes que vale la pena explorar en otro momento, una invitación abierta para que otros investigadores se sumen al diseño de un modelo de gestión de calidad orientado a la parte educativa, considerando la nueva realidad virtual, que incluyan indicadores cualitativos y cuantitativos que permitan medir la calidad educativa de las universidades públicas de México y establecer una verdadera cultura de mejora continua, quizás considerando los Objetivos de Desarrollo Sostenible de la Agenda 2030.

Finalmente, considerar explorar las TIC y otras herramientas como el big data, la inteligencia artificial y la industria 4.0 para mejorar la calidad educativa en las instituciones de educación superior, no solo de México, sino de cualquier región del mundo, aprovechando el incremento de tecnologías como apoyo para la impartición de clases en el sistema educativo, y con ello influir de manera positiva en el aprendizaje de los estudiantes universitarios. 


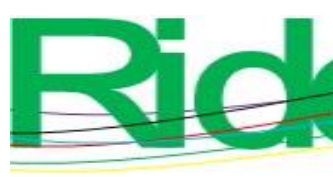

Revista Iberoamericana para la Investigación y el Desarrollo Educativo ISSN $2007-7467$

\section{Referencias}

Asamblea General. (21 de octubre de 2015). Transformar nuestro mundo: la Agenda 2030 para el Desarrollo Sostenible. Recuperado de https://www.un.org/ga/search/view_doc.asp?symbol=A/RES/70/1\&Lang=S.

Asociación Europea para la Calidad de la Educación Superior [EQNA]. (2015). Criterios y directrices para el aseguramiento de la Calidad en el Espacio Europeo de Educación $\begin{array}{llll}\text { Superior } & (E S G) . & \text { Recuperado de }\end{array}$ https://enqa.eu/indirme/esg/ESG\%20in\%20Spanish_by\%20ANECA.pdf.

Asociación Nacional de Universidades e Instituciones de Educación Superior [Anuies]. (2018). Visión y acción 2030. Propuesta de la Anuies para renovar la educación superior en México. Ciudad de México, México: Asociación Nacional de Universidades e Instituciones de Educación Superior. Recuperado de http://www.anuies.mx/media/docs/avisos/pdf/VISION_Y_ACCION_2030.pdf.

Comités Interinstitucionales para la Evaluación de la Educación Superior [Ciees]. (2018). Metodología 2018 para la evaluación y acreditación de programas educativos. Ciudad de México, México: Comités Interinstitucionales para la Evaluación de la Educación Superior. Recuperado de https://www.ciees.edu.mx/documentos/presentacionmetodologia-CIEES-2018.pdf.

Delahoz, E., Guillen, S. y Fontalvo, T. (2020). Análisis de la acreditación de calidad en programas de ingeniería industrial y los resultados en las pruebas nacionales estandarizadas, en Colombia. Formación Universitaria, 13(1), 127-134. Recuperado de https://dx.doi.org/10.4067/S0718-50062020000100127.

Ingeniería Industrial UABC. (s. f.). FCITEC de la UABC. Recuperado de http://citecuvp.tij.uabc.mx/ii/

León, C., Menéndez, A., Rodríguez, I., López, B., García, C. y Fernández, S. (2018). Importancia de un sistema de gestión de la calidad en la Universidad de Ciencias Médicas. Archivo Médico de Camagüey, 22(6), 843-857. Recuperado de http://revistaamc.sld.cu/index.php/amc/article/view/5777.

Martínez, P. (2006). El método de estudio de caso: estrategia metodológica de la investigación científica. Pensamiento \& Gestión, (20), 165-193. Recuperado de https://www.redalyc.org/articulo.oa?id=646/64602005. 

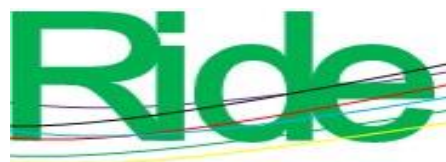

Revista Iberoamericana para la
Investigación y el Desarrollo Educativo
ISSN $2007-7467$

Moreno, I. (2018). Estrategias para la integración de sistemas de gestión de calidad y sistemas de gestión documental en una institución de educación superior. Signos, 10(1), 113125. Recuperado de https://www.redalyc.org/journal/5604/560459732006/html/.

Organización para la Cooperación y el Desarrollo Económicos (2019). Educación superior en México. Resultados y relevancia para el mercado laboral. París, Francia: Organización para la Cooperación y el Desarrollo Económicos. Recuperado de https://www.oecd.org/centrodemexico/medios/educacion_superior_en_mexico.pdf.

Organización para la Cooperación y el Desarrollo Económicos (2020). [fotografía]. OCDE. http://www.ocde.org/

Universidad Autónoma de Baja California [UABC]. (2019). Plan de Desarrollo Institucional 2019-2023. Mexicali, México: Universidad Autónoma de Baja California. Recuperado de http://www.uabc.mx/planeacion/pdi/2019-2023/PDI_2019-2023.pdf. 